\title{
Geometry of the Standard Model
}

May 21, 2008

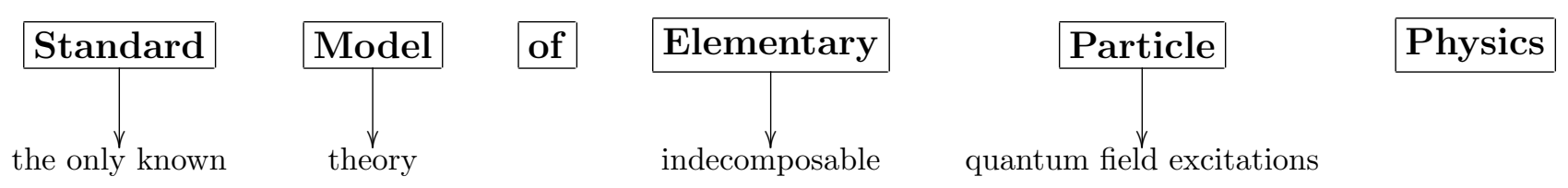

Warning! Notations and conventions used are a mixture of those found in the physics literature with those from the mathematics literature; for example in the former, the gauge fields $A_{\mu}$ usually have a different normalization which causes the coupling constants $g$ to appear in different places than here. Also the standard model is usually not formulated on Euclidean spacetime (as here), but on Minkowski spacetime, which leads to subtle differences in the fermion sector.

Furthermore, the question on how to implement neutrino masses is not quite settled; in particular, it is unknown whether the seesaw mechanism exists in reality. This is why the standard model usually does not include neutrino masses. However the presentation here is adapted to the noncommutative standard model.

\section{Contents}

1 Overview 1

2 The Fermion sector 2

3 The Yang-Mills sector 3

4 The Higgs sector

5 Fermion mass sector $\quad 6$

\section{Overview}

The Standard Model (SM) currently is the most foundational theory of physics as it is concerned with those pieces of matter that are (to current knowledge) not composite. The noncommutative standard model (NCSM) is an unquantized reformulation of the field content and the action of the SM in terms of noncommutative geometry. These notes discuss the unquantized ("classical") version of the SM, i.e. its field content and its action, with a view towards its reformulation as the NCSM. It is classical in the same sense as Maxwell's equations are a classical theory of electrodynamics - no quantum effects are included in the theory at all. This also means that the fields are in no way related to the wave functions known from quantum mechanics of point particles.

Particles and Fields. A species of particles is described in terms of a field. A field configuration is a map (say, smooth)

$$
\phi: \mathbb{R}^{4} \longrightarrow T
$$

from spacetime to some target space $T$. More generally, a field configuration is a section of a fiber bundle over spacetime. The dynamics of the field are specified by an action functional

$$
S: \phi \longrightarrow S[\phi] \in \mathbb{R}
$$


Usually the action is local in the sense that there is a Lagrangian $\mathcal{L}(\phi(x), \partial \phi(x))$ such that

$$
S[\phi]=\int d^{4} x \mathcal{L}(\phi, \partial \phi)
$$

The action functional determines the physics in one of the following ways:

\begin{tabular}{|c|c|c|}
\hline classical & quantum & Euclidean quantum \\
\hline equations of motion: & Feynman functional integral: & Wick-rotated functional integral: \\
$\delta S=0$ & $\int D \phi e^{i S[\phi]}$ & $\int D \phi e^{-S_{E}[\phi]}$ \\
\hline
\end{tabular}

Role of the Noncommutative Standard Model (NCSM). The NCSM is a reformulation of the set of field configurations together with the action functional in the unphysical Euclidean (not Minkowski space) setting. It subsumes all fields of the SM model in a spectral triple and highly reduces the arbitrariness seemingly present in the SM. It automatically includes gravity, which the SM does not. However, formulating a functional integral has not succeeded yet; therefore it is at most a classical theory.

\section{Field content of the SM.}

\begin{tabular}{|l|c|}
\hline & in NCSM encoded via \\
\hline \hline 48 Fermions (comprise matter): $24+24$ Weyl spinor fields & $\mathcal{H}$ \\
\hline 12 Gauge bosons (interactions): Yang-Mills field for $G=S U(3) \times S U(2) \times U(1)$ & $\mathcal{A}$ \\
\hline Higgs (generates masses): $\mathbb{C}^{2}$-valued scalar field & $D$ \\
\hline
\end{tabular}

\section{The Fermion sector}

Preliminaries. $\otimes$ always means $\otimes_{\mathbb{C}}$. A boldface number $\mathbf{n}$ stands for a complex Hilbert space of this dimension, say $\mathbb{C}^{n}$. The spinor representation $S$ of $\operatorname{Spin}(4)$ decomposes into two irreducible components $S=S_{L} \oplus S_{R}$ called left- and right-handed Weyl spinors, respectively. This is also reflected in the isomorphism $\operatorname{Spin}(4)=S U(2) \times S U(2)$. Each fermion species is a field with target space $S_{L}$ or $S_{R}$.

Particle content. The target space for the totality of SM fermion fields is given by

$$
\mathcal{H}_{f}=\left[S_{L} \otimes(\mathbf{3} \otimes \mathbf{2} \otimes \mathbf{3} \oplus \mathbf{2} \otimes \mathbf{3})\right] \oplus\left[S_{R} \otimes(\mathbf{3} \otimes \mathbf{2} \otimes \mathbf{3} \oplus \mathbf{2} \otimes \mathbf{3})\right]
$$

Hence we have 24 left-handed spinor fields and 24 right-handed spinor fields. Common names for these fields:

- The $\mathbf{3} \otimes \mathbf{2} \otimes \mathbf{3}$ part represents three colors $(\mathrm{r}, \mathrm{g}, \mathrm{b})$ of quark pairs coming in three generations:

$$
\left(\begin{array}{l}
u \\
d
\end{array}\right),\left(\begin{array}{c}
c \\
s
\end{array}\right),\left(\begin{array}{c}
t \\
b
\end{array}\right)
$$

up, down, charm, strange, top and bottom quark. These come in left-handed and right-handed versions.

- The $\mathbf{2} \otimes \mathbf{3}$ part represents lepton pairs also coming in three generations

$$
\left(\begin{array}{c}
\nu_{e} \\
e
\end{array}\right),\left(\begin{array}{c}
\nu_{\mu} \\
\mu
\end{array}\right),\left(\begin{array}{c}
\nu_{\tau} \\
\tau
\end{array}\right)
$$

called electron $e$ and electron neutrino $\nu_{e}$, muon $\mu$ and muon neutrino $\nu_{\mu}$, as well as tau $\tau$ and tau neutrino $\nu_{\tau}$.

Spinor algebra. The Dirac gamma matrices $\gamma_{\mu}$ generate the Clifford algebra $\mathrm{Cl}(4, \mathbb{R})$ via the relations $\left\{\gamma_{\mu}, \gamma_{\nu}\right\}=$ $2 \delta_{\mu \nu}$. These act on the spinor representation $S$ in the usual way (see [ZJ, A8.2] or [FH, 20.9]). The Dirac operator is then given by $\not \partial \equiv \gamma^{\mu} \partial_{\mu}$.

The conjugate spinor $\bar{\psi}$ is defined to be an element of the dual space $\mathcal{H}_{f}^{*}$. There is a natural antilinear map defining the assignment $\psi \mapsto \bar{\psi}$ and it is given by

$$
\mathcal{H}_{f} \rightarrow \mathcal{H}_{f}^{*}, \quad \psi \mapsto\langle\psi, \cdot\rangle
$$


Furthermore, there is a grading operator $\Gamma=\gamma_{1} \gamma_{2} \gamma_{3} \gamma_{4}$ which has $S_{L}$ and $S_{R}$ as eigenspaces with respective eigenvalues -1 and +1 . So if $\psi$ lies in the left-handed part of $\mathcal{H}_{f}$, then $\Gamma \psi=\psi$; while if $\psi$ lies in the right-handed part of $\mathcal{H}_{f}$, then $\Gamma \psi=-\psi$. Finally, there is an antilinear charge conjugation operator $C: \mathcal{H}_{f} \rightarrow \mathcal{H}_{f}$. It can be expressed in terms of the gamma matrices, but the exact form is not important. All this data corresponds to the commutative spectral triple on the spin manifold $\mathbb{R}^{4}$. The relations holding in $K O$-dimension 4

$$
C^{2}=-1, \quad C \not \partial=\not \partial C, \quad C \Gamma=\Gamma C
$$

are easy to check.

\section{Euclidean fermion action.}

$$
\mathcal{L}_{f}(\psi, \partial \psi)=\bar{\psi} C \not \partial \psi
$$

Variation with respect to $\psi$ yields the massless Dirac equation $\not \partial \psi=0$.

Motivation for gauge theory. Replace the flat connection $\partial_{\mu}$ by a fixed covariant derivative operator

$$
D_{\mu}=\partial_{\mu}-\rho\left(A_{\mu}\right), \quad \rho\left(A_{\mu}\right) \in(\mathfrak{s u}(24) \oplus \mathfrak{s u}(24))
$$

Then the new action is

$$
\mathcal{L}_{f}(\psi, \partial \psi)=\bar{\psi} C \gamma^{\mu}\left(\partial_{\mu}-\rho\left(A_{\mu}\right)\right) \psi=\bar{\psi} C \not D \psi
$$

with Dirac operator $\not D \equiv \gamma^{\mu} D_{\mu}$.

\section{The Yang-Mills sector}

Gauge theory. Gauge group $G=S U(3) \times S U(2) \times U(1)$ The 8 gauge bosons of the 8 -dimensional $S U(3)$ (called gluons) mediate the strong nuclear force which holds protons, neutrons and nuclei together. The 3 gauge bosons of $S U(2)$ (called $W^{+}, W^{-}$and $Z$ ) mediate the weak nuclear force responsible for radioactivity. The single gauge boson of the remaining $U(1)$ subgroup (the photon) is the carrier of electromagnetism ${ }^{2}$

Gauge potential: connection on (topologically trivial) principal $G$-bundle. Usually represented as a $\mathfrak{g}$-valued 1-form $A_{\mu}$ which specifies the difference to a fixed flat connection $\partial_{\mu}$. Curvature

$$
F_{\mu \nu}=\left[D_{\mu}, D_{\nu}\right]=\partial_{\mu} A_{\nu}-\partial_{\nu} A_{\mu}+\left[A_{\mu}, A_{\nu}\right]
$$

is a $\mathfrak{g}$-valued 2 -form.

\section{Yang-Mills action.}

$$
\mathcal{L}_{Y M}(A, \partial A)=-b\left(F_{\mu \nu} F^{\mu \nu}\right)=-b(F \wedge \star F)
$$

where $b$ is some bilinear form on $\mathfrak{g}$ which is invariant under the adjoint action of $G$. Since $g$ has three simple summands, the vector space of possible $b$ 's is 3-dimensional. This is commonly made more explicit in the following way: let $B_{\mu \nu}$ be the curvature of the connection corresponding to the $U(1)$ factor, and $W_{\mu \nu}$ as well as $G_{\mu \nu}$ similarly for the $S U(2)$ and $S U(3)$ factor, respectively. Then the Yang-Mills action can be written as

$$
\mathcal{L}_{Y M}=\underbrace{-\frac{1}{4 g_{s}^{2}} \operatorname{tr}\left(G_{\mu \nu} G^{\mu \nu}\right)}_{S U(3)} \underbrace{-\frac{1}{2 g^{2}} \operatorname{tr}\left(W_{\mu \nu} W^{\mu \nu}\right)}_{S U(2)} \underbrace{-\frac{1}{2 g^{\prime 2}} B_{\mu \nu} B^{\mu \nu}}_{U(1)}
$$

with real-valued coupling constants $g_{s}$ (strong force), $g^{\prime}$ and $g$ specifying the strength of the interactions compared to each other and to the fermion term (5). The factors of $-1 / 4$ and $-1 / 2$ are conventional. tr is the ordinary matrix trace, while the first term already is a number using $\mathfrak{u}(1)=\mathbb{R}$.

\footnotetext{
${ }^{1}$ Actually this group has a 6-element central subgroup acting trivially, so $G$ can effectively be replaced by $G / \mathbb{Z}_{6}$. This is important for some Grand Unified Theories like the Georgi-Glashow $S U(5)$ model.

${ }^{2}$ More accurately, the photon corresponds to a $U(1)$ subgroup lying diagonally in $S U(2) \times U(1)$. This will be discussed later.
} 
Action on the fermion sector. Representation $\rho: G \longrightarrow S U(24) \times S U(24)$ given by (see (1))

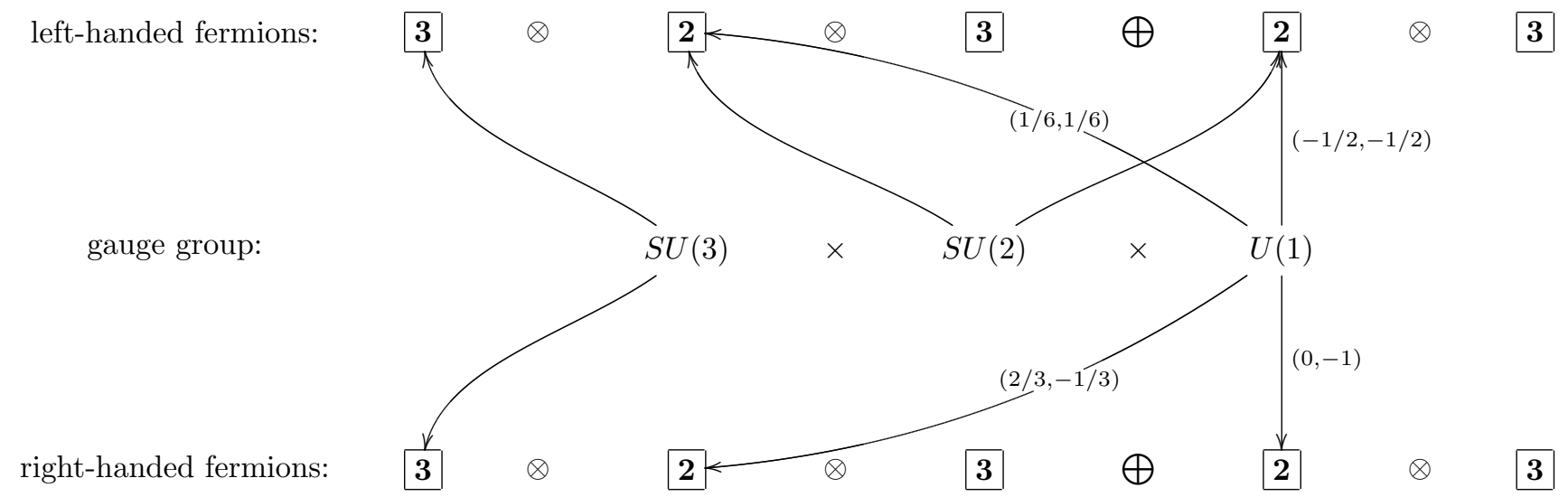

The unlabeled arrows denote fundamental representations of $S U(2)$ and $S U(3)$. The representation of $U(1) \subset \mathbb{C}$ labeled by $\left(\alpha_{1}, \alpha_{2}\right)$ is given as a diagonal matrix $z \mapsto\left(z^{\alpha_{1}}, z^{\alpha_{2}}\right)$. (Fractional values are used for historical reasons, multiply them by 6 to get an actual representation or go to the 6 -fold cover of $U(1)$.)

Note that the weak force gauge group $S U(2)$ couples only to left-handed spinors.

Gauge transformations. Gauge theories have a high degree of symmetry. For any function $\chi: \mathbb{R}^{4} \longrightarrow G$, the gauge transformation

$$
\begin{gathered}
A_{\mu} \mapsto\left[\chi A_{\mu} \chi^{-1}-\left(\partial_{\mu} \chi\right) \chi^{-1}\right] \\
\psi \mapsto \rho(\chi) \psi, \quad \bar{\psi} \mapsto \bar{\psi} \rho(\chi)^{t}
\end{gathered}
$$

leaves both Lagrangians $\mathcal{L}_{f}$ and $\mathcal{L}_{Y M}$ invariant. The behavior of $\bar{\psi}$ should be taken as a definition, which is necessary so that $\mathcal{L}_{f}$ really is invariant by $\rho(\chi)^{t} C=C \rho(\chi)^{*}$. It turned out to be an extremely successful principle to require the physical Lagrangians to be invariant under gauge transformations.

\section{The Higgs sector}

Mass terms in general. To see how masses of particles are implemented in field theory, consider an $\mathbb{R}$-valued field $\eta$ in Minkowski spacetime, i.e. signature $(+,-,-,-)$. In case of no interactions, it has a Lagrangian of the form

$$
\mathcal{L}=\frac{1}{2}\left(\partial_{\mu} \eta\right)\left(\partial^{\mu} \eta\right)-\frac{1}{2} m^{2} \eta^{2}
$$

The resulting field equation is the Klein-Gordon equation

$$
\left(\partial_{\mu} \partial^{\mu}+m^{2}\right) \eta=0
$$

which has a basis of solutions given by plane waves $\eta=e^{i p_{\mu} x^{\mu}}$ as long as the momentum vector $p_{\mu}$ satisfies

$$
-p_{\mu} p^{\mu}+m^{2}=0
$$

which is a relativistically invariant version of the Einstein energy-momentum relation $E=\sqrt{m^{2}+\vec{p}^{2}}$, as follows from $p_{\mu} p^{\mu}=E^{2}-\vec{p}^{2}$.

In Euclidean spacetime, the corresponding Lagrangian would have the form

$$
\mathcal{L}=\frac{1}{2}\left(\partial_{\mu} \eta\right)\left(\partial^{\mu} \eta\right)+\frac{1}{2} m^{2} \eta^{2}
$$

the differences being the Euclidean metric used for contracting indices and the positive sign of the mass term.

In general, the mass of the field has to be read off by comparing the mass term to the kinetic term. Example:

$$
\mathcal{L}=\frac{1}{2} \alpha\left(\partial_{\mu} \eta\right)\left(\partial^{\mu} \eta\right)+\frac{1}{2} \beta \eta^{2} \Longrightarrow m=\sqrt{\frac{\alpha}{\beta}}
$$


Gauge boson masses. Hence a mass term for a gauge boson would be a term in the Lagrangian having a form like

$$
m^{2} A_{\mu} A^{\mu}
$$

Due to the requirement of invariance under the gauge transformations (7), these are forbidden in the SM. However, experimentally one observes that the weak nuclear force has a very short range, which is due to large masses for the three gauge bosons (see [P, A1.2], also [P, 1.1.2] for the units; all other gauge bosons are observed massless)

$$
m_{W^{ \pm}} \approx 80 \mathrm{GeV}, \quad m_{Z} \approx 91 \mathrm{GeV}
$$

Consequently there is a huge theoretical problem if one wants to construct a realistic theory of particle physics without violating the gauge symmetry principle.

The Higgs field. The solution is to generate dynmical masses for these particles by a mechanism known as spontaneous symmetry breaking. One introduces an additional 2-valued field $\phi$ (the Higgs field) living in the gauge group representation $\kappa: G \longrightarrow S U(2)$ given by

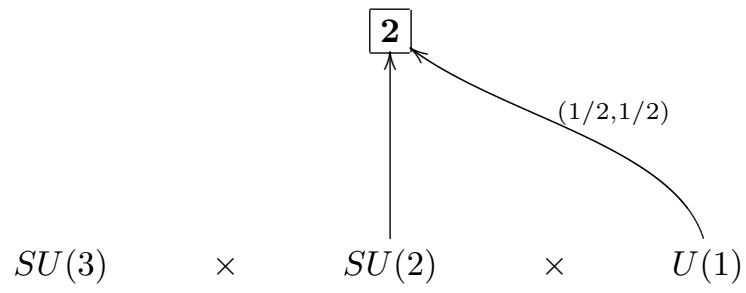

The Higgs field has the Lagrangian

$$
\mathcal{L}_{H}(\phi, \partial \phi)=\frac{1}{2}\left(D_{\mu} \phi\right)^{*}\left(D^{\mu} \phi\right)+V\left(\phi^{*} \phi\right)
$$

where ${ }^{*}$ is hermitian conjugation while $D_{\mu}=\partial_{\mu}-\kappa\left(A_{\mu}\right)$ is the covariant derivative. The potential $V$ is given by the mexican hat potential

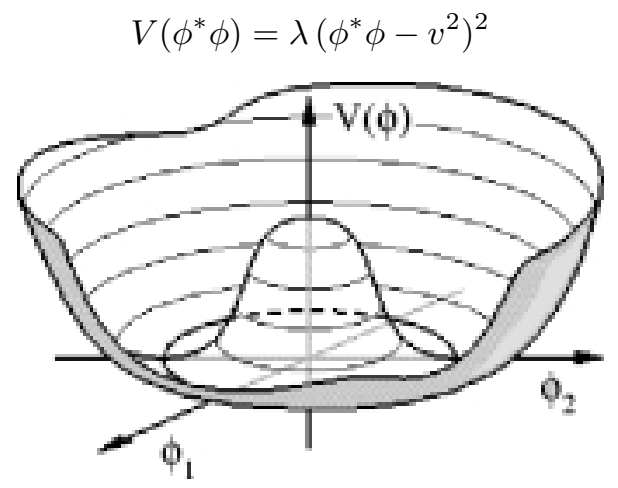

so that the Higgs field has an $S^{3}$ worth of equilibrium configurations (minima of $V$ ) at every spacetime point. If we use the term "vacuum" as synonymous to "equilibrium configuration", this means that there is a vacuum expectation value

$$
\left\langle\phi^{*}(x) \phi(x)\right\rangle=v^{2} \quad \forall x \in \mathbb{R}^{4}
$$

Gauge symmetry is broken in the sense that although the theory is gauge invariant, the vacuum will be an arbitrary one of many equilibrium configurations. So although the vacuum itself is not gauge invariant, the set of possible vacua is again gauge invariant.

Gauge boson masses and mixings from the Higgs field (GWS theory). Since $S U(2)$ acts freely and transitively on $S^{3}$ as the set of vacua. Hence by applying a unique $S U(2)$ gauge transformation we can map any Higgs field configuration to one of the form

$$
\phi(x)=\left(\begin{array}{c}
0 \\
v+h(x)
\end{array}\right)
$$


In other words, radial excitations of $\phi$ described by $h(x)$ are physical and yield the Higgs particle, while the tangential degrees of freedom are pure gauge and essentially unphysical.

Let $\left\{Y, T_{0}, T_{+}, T_{-}\right\}$be the basis of the Lie algebra given by $Y$ as the generator of $U(1)$, and

$$
T_{0}=\left(\begin{array}{cc}
i / 2 & 0 \\
0 & -i / 2
\end{array}\right), \quad T_{1}=\left(\begin{array}{cc}
0 & 1 / 2 \\
-1 / 2 & 0
\end{array}\right), \quad T_{2}=\left(\begin{array}{cc}
0 & i / 2 \\
i / 2 & 0
\end{array}\right)
$$

as the generators of $S U(2)$.

Then we can decompose functions with values in the Lie algebra $s u(2) \times u(1)$ as

$$
B(x)\left(T_{0}+Y\right)+Z(x)\left(T_{0}-Y\right)+W^{1}(x) T_{1}+W^{2}(x) T_{2}
$$

with real-valued fields $B, Z, W^{+}$and $W^{-}$. In particular, the $\mathfrak{s u}(2) \times \mathfrak{u}(1)$ part of any $G$-connection can be written as

$$
A_{\mu}=B_{\mu}\left(T_{0}+Y\right)+Z_{\mu}\left(T_{0}-Y\right)+W_{\mu}^{1} T_{1}+W_{\mu}^{2} T_{2}
$$

with 1-forms $B_{\mu}, Z_{\mu}, W_{1}$ and $W_{2}$ (dependence on spacetime point $x$ suppressed). Now we use $D_{\mu}=\partial_{\mu}-\kappa\left(A_{\mu}\right)$ to multiply out the first term of 10 . This yields one term quadratic in the gauge fields, and it is given by

$$
\frac{1}{2}\left(\kappa\left(A_{\mu}\right) \phi\right)^{*}\left(\kappa\left(A^{\mu}\right) \phi\right)
$$

Now assume that the appropriate gauge transformation was done which brought $\phi$ into the form (11). Then the contributions independent of $h(x)$ yield the following mass term for the gauge bosons:

$$
\frac{1}{2}\left[\kappa\left(A_{\mu}\right)\left(\begin{array}{l}
0 \\
v
\end{array}\right)\right]^{*}\left[\kappa\left(A^{\mu}\right)\left(\begin{array}{l}
0 \\
v
\end{array}\right)\right]
$$

Looking at the form of the generators and the representation $\kappa$, we see that $T_{0}+Y$ operates trivially so that $B_{\mu}$ drops out. What remains is

$$
\frac{1}{2}\left[-i Z_{\mu}\left(\begin{array}{l}
0 \\
v
\end{array}\right)+\frac{1}{2} W_{\mu}^{1}\left(\begin{array}{c}
0 \\
v
\end{array}\right)+\frac{i}{2} W_{\mu}^{2}\left(\begin{array}{l}
0 \\
v
\end{array}\right)\right]^{*}\left[-i Z^{\mu}\left(\begin{array}{c}
0 \\
v
\end{array}\right)+\frac{1}{2} W^{1, \mu}\left(\begin{array}{l}
0 \\
v
\end{array}\right)+\frac{i}{2} W^{2, \mu}\left(\begin{array}{l}
0 \\
v
\end{array}\right)\right]
$$

Further evaluation gives

$$
\frac{v^{2}}{2} Z_{\mu} Z^{\mu}+\frac{v^{2}}{8} W_{\mu}^{1} W^{1, \mu}+\frac{v^{2}}{8} W_{\mu}^{2} W^{2, \mu}
$$

These terms are interpreted as mass terms for the $Z$ particle as well as the $W^{ \pm}$particles (the $W^{+}$and $W^{-}$are certain linear combinations of $W^{1}$ and $W^{2}$ ). The photon described by the field $B_{\mu}$ remains massless, and note that its generator $T_{0}+Y$ lies diagonally in the $\mathfrak{s u}(2) \times \mathfrak{u}(1)$ Lie algebra. Since the Higgs field lives in the trivial representation of the $S U(3)$ component of $G$, also the gluons do not receive mass terms in this construction.

To determine the actual values of the masses, we have to compare to the kinetic terms coming from (6) as it was done in $(9)^{3}$. The result is

$$
m_{W^{ \pm}}=\frac{v}{\sqrt{2}} \cdot g, \quad m_{Z}=\frac{v}{\sqrt{2}} \cdot \sqrt{g^{2}+g^{\prime 2}}
$$

Often one defines the Weinberg angle $\theta_{w}$ by $\tan \theta_{w}=g^{\prime} / g$, where $g$ and $g^{\prime}$ can be experimentally measured by means not involving the masses of the gauge bosons. Then the Higgs field model makes the prediction that

$$
\frac{m_{W^{ \pm}}}{m_{Z}}=\cos \theta_{w}
$$

which is verified by experiment.

\section{Fermion mass sector}

$K O$-dimension. For the following to work, it is required that we assume $K O$-dimension 2 , so that in contrast to dimension 4 we have

$$
C \Gamma=-\Gamma C
$$

This is so because this equation holds in physical Minkowski spacetime, where the SM is usually formulated; without this condition, transferring the SM fermion masses to Euclidean signature would not work. Hence at this stage, considering total $\mathrm{KO}$-dimension 2 seems to be an artifact of working in unphysical Euclidean spacetime.

\footnotetext{
${ }^{3}$ modulo some subtleties about mixing of the kinetic terms of $Z$ and photon, which makes a redefinition of the $Z$ field necessary.
} 
Fermion masses without the Higgs field. Experimentally one finds that essentially all fermions are also massive 4 . Consequently, we should examine the different possiblities of implementing mass terms for fermions. In general, a mass term is a term in the Lagrangian which is quadratic in the fields $\left(\psi \in \mathcal{H}_{f}\right)$ and hence has the form

$$
\mathcal{L}_{m}(\psi)=\bar{\psi} M \psi
$$

where $M: \mathcal{H}_{f} \rightarrow \mathcal{H}_{f}$ is some fixed linear or antilinear operator. Furthermore, it has to be compatible with all symmetries of the SM, meaning that it should be invariant under the Spin(4) group as well as under gauge transformations. By (8), these translate into the requirements that $M$ has the following commutation properties with the groups acting:

$$
M \lambda=\lambda M \quad \forall \lambda \in \operatorname{Spin}(4), \quad M \rho(g)=\rho(g)^{c} M \quad \forall g \in G
$$

where ${ }^{c}$ means complex conjugate. Finally, $\mathcal{L}_{m}$ should be real-valued.

Then the set of admissible $M$ forms a real vector space which has to be determined by representation theory. By the second commutativity condition, $M$ has to be antilinear on this part of $\mathcal{H}_{f}$ where $G$ acts nontrivially. But then $C M$ is linear, so that by Schur's lemma for $\operatorname{Spin}(4)$ we can assume that $C M$ preserves handedness. It follows from (12) that $M$ itself then switches handedness. Finally this is not possible because the $S U(2)$ gauge group only acts on the left-handed sector.

Consequently, we can have mass terms only for that part of the fermion sector on which $G$ acts completely trivially. This part is given by the subspace of right-handed neutrinos $\nu_{e}, \nu_{\mu}, \nu_{\tau}$. Hence these can possibly have a Majorana mass term

$$
\mathcal{L}_{\text {Maj }}=\left(\begin{array}{lll}
\overline{\nu_{e, R}} & \overline{\nu_{\mu, R}} & \overline{\nu_{\tau, R}}
\end{array}\right) \mu\left(\begin{array}{c}
\nu_{e, R} \\
\nu_{\mu, R} \\
\nu_{\tau, R}
\end{array}\right)
$$

with a hermitian matrix $\mu$.

Yukawa couplings. Like for the gauge bosons, it is a huge problem how to write down mass terms for the fermions besides the right-handed neutrinos. Again the solution is to use terms that involve the Higgs field $\phi$, which generates mass by its nontrivial vacuum expectation value. The only possibility to do this is to use trilinear "Yukawa" couplings

$$
\mathcal{L}_{\text {Yuk }}=\bar{\psi} \cdot \phi \cdot \psi
$$

where the dots stand for insertions of linear operators whose possible form has yet to be determined. (Monomial terms with higher powers in the fields are forbidden due to renormalizability constraints in the quantized SM.) Again the operators can be linear or antilinear and have to satisfy commutativity conditions analogous to [13]. Finally we can use (11) again to obtain the actual mass terms.

Since the $S U(3)$ gauge group only acts on the quarks and neither on leptons nor the Higgs field, the Yukawa couplings are not allowed to mix these two sectors. Hence we will consider the two cases separately.

Quark masses. In order to become invariant under the $S U(2)$ gauge group, we necessarily have to mix left-handed with right-handed quarks so that the left-handed part contracts with the Higgs field in an $S U(2)$-invariant way. Since the $U(1)$ gauge group acts differently on the right-handed up-type quarks $U=\left(\begin{array}{lll}u_{R} & c_{R} & t_{R}\end{array}\right)$ and on the down-type quarks $D=\left(\begin{array}{lll}d_{R} & s_{R} & b_{R}\end{array}\right)$ (see 2 , we can also consider these two separately. Thus we have reduced the possible mass terms to the following type:

$$
\mathcal{L}_{\text {Yuk }, q}=\overline{Q_{L}} \alpha_{u} \phi \mathcal{M}_{u} U_{R}+\overline{Q_{L}} \alpha_{d} \phi \mathcal{M}_{d} D_{R}+\text { h.c. }
$$

where $Q_{L}$ stands for all left-handed quark fields, $\alpha_{u}, \alpha_{d}: \mathbf{2} \rightarrow \mathbf{2}$ are linear or antilinear, and

$$
\mathcal{M}_{u}, \mathcal{M}_{d}: S_{R} \otimes \mathbf{3} \otimes \mathbf{3} \rightarrow S_{R} \otimes \mathbf{3} \otimes \mathbf{3}
$$

are also linear or antilinear. "h.c." means "hermitian conjugate", and stands for the same two terms with handedness reversed and complex conjugates taken such that the whole expression is real-valued.

Since the left-handed and right-handed subspaces of $\mathcal{H}_{f}$ are orthogonal, we necessarily need to have a charge conjugation $C$ in $\mathcal{M}_{u}$ and $\mathcal{M}_{d}$, which is (as above by (12) and Schur's lemma) the only operator reversing handedness

\footnotetext{
${ }^{4}$ Although neutrinos are extremely light, and the $u$ quark might potentially be completely massless.
} 
and commuting with the spin group. Also by Schur's lemma, $\mathcal{M}_{u}$ and $\mathcal{M}_{d}$ have to operate trivially on color space. Hence all that is possible is

$$
\mathcal{M}_{u}=C M_{u}, \quad \mathcal{M}_{d}=C M_{d}
$$

with $M_{u}$ and $M_{d}$ both $3 \times 3$ matrices operating on generation space.

As for $\alpha_{u}$ and $\alpha_{d}$, by Schur's lemma there can at most be one linear and one antilinear operator such that the expression is $S U(2)$ invariant (recall (8)). It is not hard to check that the two possibilities

$$
. c \text { (complex conjugation on } \mathbf{2}), \quad\left(\begin{array}{cc}
0 & 1 \\
-1 & 0
\end{array}\right)
$$

work; the first corresponds to the usual hermitian inner product on $\mathbf{2}$, while the second corresponds to the usual volume form on 2 . If one takes the second operator as $\alpha_{u}$ and the first one as $\alpha_{d}$, then invariance under the remaining $U(1)$ gauge group also holds, which follows from the calculations

$$
\begin{aligned}
& \frac{1}{6}+\frac{1}{2}-\frac{2}{3}=0 \\
& \frac{1}{6}-\frac{1}{2}+\frac{1}{3}=0
\end{aligned}
$$

where the first summand represents the transformation properties of $\bar{Q}_{L}$, the second summand the one of $\phi$ (resp. its complex conjugate), and the third summand the one of the complex conjugate of $U_{R}$ and $D_{R}$ (recall the $C$ contained in $\mathcal{M}_{u}$ and $\mathcal{M}_{d}$ ).

Using the vacuum expectation value

$$
\phi=\left(\begin{array}{l}
0 \\
v
\end{array}\right)
$$

we finally end up with the mass terms

$$
\mathcal{L}_{\text {Yuk }, q}=\overline{U_{L}} C M_{u} U_{R}+\overline{D_{L}} C M_{d} D_{R}
$$

In contrast to the Majorana mass terms, these do contain a charge conjugation operator; expressions like this one are Dirac mass terms 5

Quark mixing. We can gain some insight into the phenomenology of this by taking a singular value decomposition

$$
M_{u}=X^{*} M_{u}^{\prime} Y
$$

with unitaries $X, Y$ and positive semidefinite diagonal

$$
M_{u}^{\prime}=\operatorname{diag}\left(m_{u}, m_{c}, m_{t}\right)
$$

Then a redefinition of the quark fields as

$$
U_{R} \mapsto Y^{*} U_{R}, \quad U_{L} \mapsto X^{c} U_{L}
$$

yields the new mass terms

$$
\mathcal{L}_{\text {Yuk }, q}=\bar{u} C m_{u} u+\bar{c} C m_{c} c+\bar{t} C m_{t} t+\overline{D_{L}} C M_{d} D_{R}+\text { h.c. }
$$

This means that we can choose the up-type quarks to be their own mass eigenstates. Of course we can do the same thing with the down-type quarks also. However by the $S U(2)$ gauge group action, each up-type quark is paired with a down-type quark; hence we should not expect that the $S U(2)$ partners of the up-type quark mass eigenstates are exactly the mass eigenstates of the down-type quarks. Instead, there should be unitary transformation going from mass eigenstates $d, s, b$ to weak eigenstates $d^{\prime}, s^{\prime}, b^{\prime}$, where the weak eigenstates are defined to be the $S U(2)$ partners of the up-type quark mass eigenstates. This unitary matrix is generally known as the CKM matrix

$$
V=\left(\begin{array}{ccc}
V_{u d} & V_{u s} & V_{u b} \\
V_{c d} & V_{c s} & V_{c b} \\
V_{t d} & V_{t s} & V_{t b}
\end{array}\right)
$$

Experimentally measuring the entries of this matrix ${ }^{6}$ and checking this for unitarity is a good test of the assumption of 3 generations in the SM; detecting non-unitarity would imply the existence of other as yet unknown quarks.

\footnotetext{
${ }^{5}$ Warning: in Minkowski spaces this is exactly opposite.

${ }^{6}$ Actually there is still freedom left to redefine the fields, so one can only measure certain combinations of these parameters.
} 
Lepton masses. In exactly the same way, one can classify Yukawa couplings for the lepton sector. This yields mass terms for the electron, muon and tau particles as well as additional masses for the neutrinos. Also using a singular value decomposition, one can turn the electron, muon and tau lepton into lepton mass eigenstates, so that the Lagrangian has the form

$$
\mathcal{L}_{\text {Yuk }, l}=\bar{e} C m_{e} e+\bar{\mu} C m_{\mu} \mu+\bar{\tau} C m_{\tau} \tau+\overline{\nu_{L}} C M_{\nu} \nu_{R}+\text { h.c. }
$$

Neutrino mixing and the seesaw mechanism. We can summarize the neutrino mass terms as the following

$$
\left(\begin{array}{ll}
\overline{\nu_{L}} & \overline{\nu_{R}}
\end{array}\right)\left(\begin{array}{cc}
0 & C M_{\nu} \\
M_{\nu}^{*} C^{*} & \mu
\end{array}\right)\left(\begin{array}{c}
\nu_{L} \\
\nu_{R}
\end{array}\right)
$$

Then the masses of the neutrinos are given as the eigenvalues of this large matrix. Experimentally, it is observed that neutrinos are extremely light particles (though not massless), with rest energy way below the energy of a single photon in the visible spectrum. This can be explained in the seesaw mechanism by assuming that the entries of $M_{\mu}$ have the same order of magnitude as $m_{e}, m_{\mu}, m_{\tau}$, while the entries of $\mu$ are extremely large. Then we can regard the matrix as a small perturbation of the matrix

$$
\left(\begin{array}{ll}
0 & 0 \\
0 & \mu
\end{array}\right)
$$

which has three vanishing and three generically large eigenvalues. The eigenvalues of the total matrix can then be approximately determined in second-order perturbation theory for linear operators [CT, 11.2.2]. As one would intuitively expect, one obtains three large eigenvalues and three very small eigenvalues, where the former are interpreted as belonging to very massive neutrinos which have not been detected yet due to their large mass, while the latter are interpreted as the ordinary neutrinos which physicists currently can detect.

For a simple explicit calculation, assume one neutrino generation instead of three, forget about $C$ and assume $M_{\nu}$ real, so that we have a $2 \times 2$ mass matrix

$$
\left(\begin{array}{cc}
0 & M_{\nu} \\
M_{\nu} & \mu
\end{array}\right)
$$

The eigenvalues of this matrix are given by

$$
\lambda_{ \pm}=\frac{\mu \pm \sqrt{\mu^{2}+4 M_{\nu}^{2}}}{2} \approx \frac{\mu \pm\left(\mu+2 M_{\mu}^{2} / \mu\right)}{2}
$$

yielding a large mass $\lambda_{+}$around $\mu$, and a small mass $\lambda_{-} \approx-M_{\nu} / \mu^{2}$.

\section{References}

[P] Donald H. Perkins, Introduction to High Energy Physics.

[PS] Michael E. Peskin, Daniel V. Schroeder: An Introduction to Quantum Field Theory.

[ZJ] Jean Zinn-Justin: Quantum Field Theory and Critical Phenomena.

[FH] William Fulton, Joe Harris: Representation Theory.

[CT] Claude Cohen-Tannoudji, Bernard Diu, Franck Laloë: Quantenmechanik Teil 2. 